\section{Warm up}

Climbing the walls, losing our way, and bouncing for fun. In this issue we bring you new sports, new injuries, and new fields of research. Indoor rock climbing sounds like a contradiction in terms but what started off as a training exercise to prepare climbers for the great outdoors has now become a sport in itself. What was a lonely, thoughtful challenge against the natural world has become an athletic event promoted by television with all the glamour of show business. The long calculated mountain ascent has become a short sprint with a completely different physiological load (p 224). Orienteering is not a new sport either, but, similarly, it has evolved from a recreational map reading exercise to become a competitive athletic event with its own pattern of injury (p 205). Some sports are completely new, such as aeroball, synthetic, manufactured, and only possible because of new equipment and facilities within leisure centres. No wonder that it has led to a completely different pattern of injury ( $p 200$ ). These new sports lead us to think in different ways about injury. We also publish a paper which suggests a novel reason to take exercise. Many believe that exercise improves the way they think, but now we can point to research evidence showing that exercise itself enhances creativity (p 240). When this becomes widely known you may soon have to take your place in the leisure centre queue behind lines of bearded academics, artists, and writers.

\title{
Setting standards, chasing goals, and measuring performance
}

Nothing quite matches the excitement of the new season, the anticipation, the first few games, watching the players, the managers, and the teams moulding together. It appeals to us all because we understand sport. We recognise the importance of team work, having common goals, keeping it simple, but still allowing the individual to flourish. Every player knows about playing to a common style, defining roles and responsibilities, and working together. Even the spectators can anticipate how a team is going to play, and how a manager will react to certain events. Each successful team has an identifiable style and we know that a team that lacks that cohesion, a common purpose, or the will to improve will fail, irrespective of the number of talented individuals they field.

In sports medicine the principles are the same, it is just that some of the words are different. As a specialty, however, we do not appear to have common goals, a cohesive strategy, a recognisable ethos, or effective leadership. Neither the public nor our colleagues in other branches of the profession know what kind of service we offer. Just as in sport, we cannot change it all overnight, but we can identify some key areas in which to begin.

If, as a specialty, we were asked to define our core values and indicate desirable standards of care we would have some difficulty. There are no accepted clinical standards in sports medicine and no established quality measures. It is unclear to whom we are accountable, who should define standards of care, or who should police them. Basic injuries may be treated differently depending on where in the world you live, the medical care system, and what specialist you attend. Locally, nationally, and internationally we see very different approaches to clinical examination and treatment with little agreement on appropriate management. There are no core standards or guidelines, and treatments vary greatly from country to country. Clinicians may act with the best intentions but there must be a better way.

We need to define some baseline standards in prevention, treatment, and organisation and having defined the baseline, we can then seek improvement and raise standards. This type of quality assurance gives the public confidence in our ability to deliver high quality care. Public endorsement by a big name athlete, or association with a high profile team, are no indication of quality care, but in the absence of any other quality measures these are the cri- teria the public will use. If we can begin to put our own house in order we can then move on to consider some of the opportunities for collaboration available at an international level. Our association has increasing international links and close relations with our colleagues in many countries and it may be possible to reach some international consensus on key issues.

Education is the major purpose of BASM. There is a particular need to look at standards in education. New sports medicine qualifications-diplomas, masters programmes, and certificates-are increasingly available, but there is always the anxiety that exams and courses become simply a money making exercise and lose sight of their main educational purpose. There are no common entry qualifications, no core curricula, and no recognised examination standards. We cannot hope to move towards common standards or criteria referenced examinations until we can at least agree on entry criteria. Some are open to medical graduates only, some to those with physiotherapy qualifications, and some to both. Multidisciplinary education has many benefits but surely the educational needs of general practitioners, orthopaedic trainees, and physiotherapists are different. We cannot rank an MSc or a Diploma and we do not know what training graduates have had or what they are qualified to do. While it is laudable that people with vision and drive have created these educational opportunities, it is impossible to know the value of each qualification. The first steps should be to standardise entry procedures, establish a core curriculum, and define common objectives. Currently there is chaos, but it is a creative, energetic, and very positive chaos that just needs some structure and direction. There is no wish to strangle this tremendous growth, just to nudge it in the right direction.

Every manager looks at the results at the end of the season as they provide a clear indication of performance. He or she assesses the team's performance in detail, corrects the weak points, builds on the strengths, and plans a new strategy. Each new season offers the challenge to improve on last seasons results. Somehow when you translate these principles of sport into medicine and call them audit, quality assurance, or clinical standards, they may not seem quite the same, but they are.

DOMHNALI MACAULEY 